\title{
Anabases
}

ANABASES Traditions et réceptions de l'Antiquité

$14 \mid 2011$

Varia

\section{La " cérémonie » comme catégorie critique dans le Théâtre des Grecs du P. Brumoy}

Jean-Philippe Grosperrin

\section{(2) OpenEdition}

\section{Journals}

Édition électronique

URL : http://journals.openedition.org/anabases/2153

DOI : 10.4000/anabases.2153

ISSN : 2256-9421

Éditeur

E.R.A.S.M.E.

Édition imprimée

Date de publication : 1 octobre 2011

Pagination : 61-73

ISSN : 1774-4296

\section{Référence électronique}

Jean-Philippe Grosperrin, «La « cérémonie » comme catégorie critique dans le Théâtre des Grecs du P. Brumoy », Anabases [En ligne], 14 | 2011, mis en ligne le 01 octobre 2014, consulté le 20 octobre 2019. URL : http://journals.openedition.org/anabases/2153 ; DOI : 10.4000/anabases.2153

(c) Anabases 
Anabases 14 (2011), p. 61-73.

\section{La " cérémonie " comme catégorie critique dans le Théâtre des Grecs du P. Brumoy}

JeAn-PhiLIPpe Grosperrin

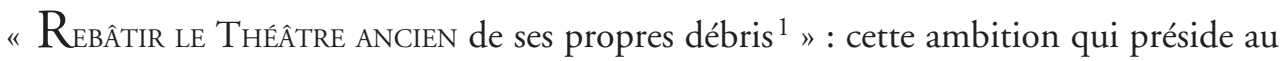
Théatre des Grecs du P. Brumoy ne signifie ni une traduction systématique du corpus ni une poétique adoptant la forme du traité :

"On a donné beaucoup à la théorie sur les traces d'Aristote, et même à la pratique, comme M. l'abbé d'Aubignac. Il y manquait d'exposer le Théâtre ancien dans le point où il faut l'envisager pour le bien connaitre, c'est-à-dire en lui-même par l'exposition des œuvres Tragiques et Comiques ${ }^{2}$."

Dans une lettre contemporaine au marquis de Caumont, Brumoy précise la façon dont s'articulent la forme de l'anthologie et la perspective critique : l'auteur déclare avoir eu en vue " une espèce de poétique par les faits ", où " la confrontation des quatre poètes entre eux, avec Sénèque et avec nos plus illustres modernes, servira enfin, suivant mon projet, à faire connaître les uns par les autres, et à former un tout de diverses parties, pour mettre le lecteur en état de prononcer $^{3}$ ".

Ce refus du dogmatisme est du reste revendiqué dans le corps de l'ouvrage, où le commentateur entend développer l'examen des anciens Grecs en se gardant de l'ad-

1 P. Brumoy, Théâtre des Grecs, Paris, Rollin, 1730, "Discours sur le Théâtre des Grecs ", t. I, p. xiv. Toutes les références seront données d'après cette édition originale en 3 volumes in $-4^{\circ}$. L'orthographe a été modernisée, mais l'usage des majuscules est strictement respecté.

2 Ibid., p. xxvij.

3 Lettres du P. Brumoy au marquis de Caumont, éd. J.-M. Prat, Paris, Julien, 1857, p. 25. 
miration fanatique comme du mépris moderne pour des formes et un esprit éloignés de la civilisation perfectionnée. Brumoy redéfinit l'argument du " progrès de l'esprit humain ${ }^{4}$ » qu'avaient brandi les Modernes dans la Querelle pour répudier les modèles antiques 5 . D'une part, si « la belle Antiquité » a pour " caractère général » un " caractère simple et noble par sa simplicité même ${ }^{6}$ ", cette simplicité est présentée comme le résultat d'une continuité historique : Racine est à Rotrou ce qu'Euripide est à Eschyle ${ }^{7}$, et l'examen en parallèle des Iphigénie en Aulide grecque et française en souligne les convergences ${ }^{8}$. D'autre part, si la tragédie moderne peut atteindre la grandeur par les voies de la simplicité, c'est aussi dans la mesure où « les Muses françaises » ont bénéfice à " ne pas perdre de vue les modèles grecs » et à " ne pas dédaigner un Théâtre qui a pour mère la nature, pour âme les passions, pour art la simplicité : Théâtre peut-être inférieur au nôtre en dignité et en noblesse ; mais supérieur en simplicité et en bienséance ${ }^{9}$ ".

Cependant, la modélisation de la simplicité grecque ne se fait pas sans hésitations, ou plus exactement elle ne se fait pas sans faire apparaittre une relation constante entre le naturel de l'expression et l'appareil de la magnificence. En développant le parallèle de la tragédie grecque et de la moderne, Brumoy écrit :

"Le caractère singulier qui perce à travers ces mœurs antiques [...] n'est véritablement pas cette noblesse, cette pompe, cette magnificence élégante et recherchée des sentiments de notre Théâtre. On y voit tout cela, mais réduit aux bornes de la simple nature, et dépouillé de cet éclat qui est propre aux Monarchies ${ }^{10}$."

De ce point de vue, la tragédie grecque se signalerait par un assujettissement de la pompe au naturel. Mais au même moment, et conformément à la nostalgie exprimée par les théoriciens du XVII ${ }^{\mathrm{e}}$ siècle pour les représentations antiques ${ }^{11}$, Brumoy en célèbre la «magnificence » inégalée : "Les changements de décorations, les vols, les Gloires, et tout ce qu'étalent les Théâtres d'Europe y était employé, mais avec plus de dépense et de grandeur ${ }^{12}$. » Aussi déplore-t-il l'abandon des chœurs comme « la perte de la partie

$4 \quad$ Théatre des Grecs, t. I, p. 397.

5 Voir J. Dagen, L'Histoire de l'esprit humain dans la pensée française, de Fontenelle à Condorcet, Paris, Klincksieck, 1977, chap. III.

6 Théâtre des Grecs, "Discours sur le parallèle du Théâtre ancien et du moderne ", xxI, t. I, p. clij.

$7 \quad$ Ibid., "Conclusion générale ", t. III, p. 316.

8 Voir É. Flamarion, "Les "beautés de Racine" selon le P. Brumoy ", Littératures classiques 52 (2004), p. 75-86.

9 Théâtre des Grecs, "Conclusion générale », t. III, p. 318.

10 Ibid., "Discours sur le parallèle du Théâtre ancien et du moderne », XXI, t. I, p. clj.

11 Voir J. de La Mesnardière, La Poétique, Paris, A. de Sommaville, 1640, p. 431 ; Fr. D'Aubignac, La Pratique du théâtre [1657], éd. H. Baby, Paris, Champion, 2001, p. 485 ; A. DACIER, L'Edipe et l'Électre de Sophocle. Tragédies grecques traduites en français avec des Remarques, Paris, Cl. Barbin, 1692, p. 152.

12 Théâtre des Grecs, "Discours sur l'origine de la tragédie ", XVIII, t. I, p. xcvj. 
la plus pompeuse du spectacle ${ }^{13}$ " : on y perd à la fois "la continuité de l'action, et un spectacle magnifique qui sert à la soutenir, et qui est, pour ainsi dire, le fond ou l'accompagnement du tableau ${ }^{14}$ ".

Dans ces conditions, il n'est pas inutile peut-être d'observer la place que tient chez Brumoy la catégorie de la cérémonie, qui caractérise la dramaturgie de la tragédie grecque en même temps qu'elle touche, sous l'Ancien Régime, aux valeurs de la pompe et de la magnificence. Si l'on se rappelle que les poétiques du classicisme interprètent la définition aristotélicienne de la tragédie (action noble, style grave) en y intégrant expressément la magnificence du spectacle ${ }^{15}$, cela revient à ranger la tragédie offerte aux yeux du public sous le signe de la cérémonie, définie par le Dictionnaire de Trévoux en 1732 en ces termes : "Assemblage de plusieurs actions, formalités, et manières d'agir, qui servent à rendre une chose plus magnifique et plus solennelle. Solennes ritus, ceremonia. Les entrées des Rois se font avec grande cérémonie. [...] on leur érige des trônes, des arcs de triomphe ${ }^{16}$. " On se rappellera justement combien le théâtre scolaire des jésuites a particulièrement veillé aux représentations de la souveraineté royale. Mais de surcroît la notion de cérémonie intéresse la religion : « Cérémonie, dans les choses ecclésiastiques, est proprement une action extérieure établie par l'Église pour rendre le service divin plus auguste et plus respectable. Sacer ritus, caremonia. L'Ancien Testament était tout plein de mystères et de cérémonies, etc. ${ }^{17}$. » Et c'est à propos des " mystères " que l'institution du rite est définie pour " les fausses Religions " : "Ces secrets de la Religion étaient appelés des mystères, non parce qu'ils étaient incompréhensibles, ni élevés au-dessus de la raison, mais seulement parce qu'ils étaient couverts et déguisés sous des types et des figures, afin d'exciter la vénération des peuples par cette obscurité, et par des cérémonies ${ }^{18}$."

On se demandera donc dans quelle mesure la représentation des cérémonies antiques qu'offraient les tragédies grecques s'accorde ici avec l'éloge d'une simplicité et d'un naturel dont la valeur est posée comme transhistorique ; c'est-à-dire aussi dans quelle mesure Brumoy intègre le rite dans la réception moderne de l'ancienne tragédie.

13 Ibid., " Discours sur le parallèle du Théâtre ancien et du moderne ", XVIII, t. I, p. cxlvj.

14 Ibid., XXIII, t. I, p. clvj-clvij.

15 Sur cet aspect, voir Chr. DeLMAS, "L'unité du genre tragique au XVII e siècle », Littératures classiques 16 (1992), p. 110-113 ; J.-Y. Vialleton, "Une catégorie "mineure" de l'esthétique théâtrale au XVII ${ }^{\mathrm{e}}$ siècle : la magnificence ", Littératures classiques 51 (2004), p. 243-245. Dans le prologue d'Atys de Lully, Melpomène célèbre ainsi " l'Appareil magnifique / De la Muse tragique / Et de ses Spectacles pompeux » (Ph. Quinault, Atys, tragédie en musique, Paris, Ballard, 1676, n.p.).

16 Dictionnaire universel français et latin, dit Dictionnaire de Trévoux, Paris, É. Ganneau, 1732, s.v. "Cérémonie ».

17 Ibid.

18 Ibid. 
Observons d'abord le commentaire des déplorations funèbres, fortement ritualisées par l'écriture lyrique et la participation du chœur. Dans Iphigénie en Tauride, le rite funéraire vaut surtout pour sa valeur d'ironie dans l'économie d'une tragédie de la reconnaissance :

"Le Chœur naturellement amené par l'ordre de la Prêtresse Iphigénie, vient la seconder dans le triste sacrifice qu'elle fait pour un frère qu'elle croit mort. Erreur charmante, qui outre le spectacle qu'elle produit, est une grande adresse pour rendre plus agréable la surprise d'Iphigénie, quand elle reverra inopinément ce frère chéri qu'elle vient de pleurer ${ }^{19}$."

Le spectacle de la célébration rituelle en présence du chœur, introduit « naturellement ", trouve sa justification dans la cohérence d'une structure pathétique, c'est-à-dire dans une économie de l'ensemble, déterminée par la péripétie de l'agnition. De même, dans l'Électre d'Euripide, la « triste cérémonie » par laquelle la protagoniste célèbre Agamemnon n'est pas glosée dans sa dimension incantatoire ou plastique, mais dans sa fonction pathétique :

«Électre se croyant seule, renouvelle ses plaintes en remplissant son urne. Ce qu'elle dit est exprimé par une espèce d'Ode, que les anciens appelaient Monodie ou Monologue en chant. [...] Elle a mis bas son urne avant que de commencer cette triste cérémonie, c'est-àdire, avant que de payer à Agamemnon le tribut de ses larmes et de ses cris lugubres ${ }^{20}$."

On est assez près alors de la conception du jésuite Rapin dans ses Réflexions sur la poétique (1675) : la qualité tragique se juge d'abord au critère des "discours quand ils sont naturels et passionnés ${ }^{21}$ ». Inversement, dans cette même tragédie, l'ancrage cérémoniel du meurtre d'Égisthe est dévalué comme contraire à un " naturel " derrière lequel se profile l'impératif de vraisemblance :

" [Chez Sophocle] Clytemnestre est tuée durant l'absence d'Égisthe, sans que le bruit de cet attentat se répande hors du Palais, dont Oreste s'est rendu le maître : et ensuite Égisthe revient d'un voyage, ainsi qu'on l'attendait, pour tomber entre les mains de son ennemi. Cela est sans doute beaucoup plus naturel que de supposer Égisthe tué au milieu de ses domestiques dans un sacrifice, et Clytemnestre attirée dans un piège assez mal

19 Théâtre des Grecs, t. II, p. 72.

20 Ibid., t. I, p. 214 (je souligne). Brumoy commente dans le même esprit la "cérémonie funèbre " des Choéphores d'Eschyle, qui vaut pour "la force \& l'énergie " des vers du Chœur, tandis que dans le dialogue les "fréquentes invocations " d'Électre aux dieux " donnent de l'âme " à la scène, et sont "l'expression pure de la vengeance $\&$ de la douleur» (ibid., p. 199 et 206).

21 R. Rapin, Réflexions sur la poétique de ce temps, éd. E. Dubois, Genève, Droz, 1970, p. 107. 
tendu. En effet la supposition des couches d'Électre n'est guère vraisemblable ${ }^{22}$. La Reine sa mère aurait-elle dû ignorer la grossesse de sa fille, et la naissance d'un petit-fils ? de plus, sur quel fondement Électre se tenait-elle assurée que sa mère aurait la complaisance de se rendre à sa chaumine au moment qu'elle en serait priée ? si pourtant une de ces mesures avait été rompue, le secret était découvert, et la conjuration manquée. On voit bien qu'Euripide a voulu donner plus de grandeur et de célébrité à l'attentat d'Oreste sur Egisthe, en choisissant un jour solennel, un sacrifice, et un sacrificateur de cette importance, qui devient lui-même victime au milieu de sa Cour et de ses sujets. Mais c'est cela même qui rend la machine trop embarrassée, et les ressorts trop compliquées ${ }^{23}$."

Aux yeux de Brumoy, la solennité tragique choisie par Euripide joue contre un fonctionnement harmonieux du dénouement. La cérémonie, tragique en elle-même, empêcherait ainsi la fluidité dramatique de l'action.

Ailleurs, la comparaison entre Sénèque et ses sources grecques, que Brumoy définit dans les discours liminaires comme " confrontation critique ", permet d'opérer à propos du sujet des Troyennes une distinction entre la déploration rituelle et un pathétique fondé sur le naturel de l'expression et des caractères. Voici par exemple comment le jésuite commente deux séquences de vers "lugubres ", avec interaction de la protagoniste Hécube et du chœur.

«L'accablement où elle est [Hécube] lui ôte la force de se lever. Relevée cependant par le secours des femmes, elle fait en vers lugubres le récit de tous ses maux. [...] Le Chœur entre dans ses sentiments. Il faut remarquer qu'il n'est pas composé des femmes du premier rang. L'on entend d'un autre côté de grands cris. Ce sont ceux des autres Dames Troyennes qui sont enfermées dans une tente, et qui voient que l'on précipite leur départ et leur esclavage. Tout le reste de l'Acte ne roule que sur leur inquiétude touchant leur destinée. C'est un deuil de femmes effrayées sans nulle ressource d'espoir, et une suite de plaintes plus naturelles que faciles à exprimer ${ }^{24}$."

«Hécube frappée de cet adieu [de Cassandre] se pâme : on la rappelle à la vie. Mais elle ne revoit la lumière que pour se peindre plus vivement l'excès de son infortune. Ce sont les plus grands traits. Et c'est toujours le même objet, la grandeur de sa fortune passée, l'ancienne gloire de Troie, ses murs renversés, ses palais en cendre, Priam tombant à l'Autel de Jupiter, ses fils et ses filles ou morts ou esclaves, elle-même enfin réduite à la plus dure captivité. Euripide entre dans le détail, et ne fait pas difficulté de dire en quoi consiste cet esclavage, à savoir à remplir les plus bas offices des domestiques. Cette peinture que fait

22 Électre «l'engage à entrer dans la cabane pour y faire le sacrifice ordinaire au dixième jour après la naissance de l'enfant »(Théâtre des Grecs, t. I, p. 228).

23 Ibid., p. 229 (je souligne).

24 Ibid., t. II, p. 575 (je souligne). Cf. Euripide, Les Troyennes, v. 99 sq. 
Hécube renouvelle la douleur des Troyennes, qui toutes font un intermède en chant lugubre, et tout rempli des mêmes idées sous des tours différents ${ }^{25}$."

Le pathétique vaut pour sa résonance humaine, pour la proximité de la représentation avec l'humanité ordinaire. Brumoy ne met nullement en relief l'apprêt lyrique de la déploration mais bien son naturel, entendu comme " ressemblance " avec des émotions dont le spectateur est susceptible. C'était la perspective énoncée dans le commentaire du début d'Hécube :

"La déplorable Reine fait éclater ici une douleur difficile à représenter. Elle est mère; c'est [Cassandre] l'unique bien que lui ait laissé la fortune, et l'on veut lui enlever ce seul bien que les Dieux avaient épargné. Hors d'elle-même, elle court çà et là. Elle appelle sa fille à grands cris. Polyxène entend ces cris, et sort de son appartement, qu'on suppose voisin. Hécube a de la peine à s'exprimer. C'est une mère désespérée ; sorte de peinture où excelle Euripide ${ }^{26}$."

Or le commentaire est très différent pour La Troade de Sénèque, dont Brumoy déplore l'absence d'unité centripète et de continuité. La grande lamentation collective de l'acte I fait ainsi l'objet d'une analyse assez équivoque :

"Hécube sans autre passage qui unisse ce qu'elle va dire avec ce qu'elle a dit, s'avise tout à coup de s'écrier : Lamenta cessant? [v. 63] "Quoi ? Nous différons notre deuil ? Compagnes de ma captivité, frappez votre sein, faites tout retentir de vos gémissements : et célébrez les funérailles de Troie." Et justa Troja facite.

Le Chœur obéit. [...] Mais on trouverait bien du mécompte dans le détail. Ici Hécube commence le deuil en cérémonie. Elle donne aux autres femmes l'exemple et le signal. Toutes de concert laissent flotter leurs cheveux, se les arrachent, se couvrent de poussière, déchirent leurs vêtements, et se battent la poitrine : le tout en cadence et avec toutes les marques du désespoir. Aussi est-ce un deuil général pour la patrie entière, et sans exemple dans les Tragédies Grecques. Il en devait coûter aux Acteurs qui jouaient de pareils rôles, si on les jouait. [...] Hécube s'en va, l'on ne sait pourquoi ${ }^{27}$. "

Brumoy est-il gêné par l'ostentation du caractère fortement cérémoniel de la déploration ou bien par sa violence ? L'expression en cérémonie signale assez nettement une forme de ruine du naturel dans l'expression du pathos, désignant sans doute un style trop apprêté, conformément aux griefs contemporains et ordinaires contre la rhétorique de Sénèque et ses " broderies ${ }^{28}$ ». Et que penser du commentaire sur la

25 Ibid., t. II, p. 577 (je souligne). Cf. Euripide, Les Troyennes, v. 463 sq.

26 Ibid., t. II, p. 358 (je souligne). Cf. Euripide, Hécube, v. 151 sq.

27 Ibid., t. II, p. 593 (je souligne).

28 Sur la réception du théâtre de Sénèque dans le classicisme français, voir Fl. de CAIGNY, Sénèque le Tragique en France (XVI'-XVII siècles) : imitation, traduction, adaptation, Paris, 
dimension "générale » du deuil, sinon qu’il impute à Sénèque un défaut de pertinence expressive en raison d'une expansion outrée de la plainte, à la fois pour l'élocution et pour l'action requises? De même avec le récit du sacrifice de Polyxène, qui pèche par excès et non par omission :

"C'est la description d'un hymen funèbre. On y voit les Dames Grecques avec Hélène, des torches à la main : on y voit Polyxène que son malheur rend encore plus belle. Mais quand ce vient au sacrifice même, Sénèque oublie la simplicité d'Euripide, et se livrant à son génie, il dit des choses étonnantes. Polyxène n'est point fière, elle est féroce : elle ne range point ses vêtements, comme le dit Euripide ; mais elle tombe avec effort, comme pour briser de courroux le tombeau d'Achille : le sang ne tombe plus sur le sépulcre pour l'arroser, mais le sépulcre le boit tout entier ${ }^{29}$."

Cependant le caractère incantatoire ou paraliturgique de la scène évoquée par la narration n'est aucunement signalé, pour autant qu'il ne connaît là aucune réalisation dans le spectacle. Question de style rhétorique, mais question aussi d'économie de l'espace scénique. De fait, la dramaturgie cérémonielle de la tragédie grecque se trouve ailleurs valorisée, et précisément dans ses aspects scénographiques, de telle sorte que la cérémonie rituelle offerte en spectacle s'impose comme objet d'un intérêt particulier, jusqu’à la fascination.

Assurément Brumoy se montre sensible à la sacralité des cérémonies représentées dans le théâtre des Grecs. On le remarque d'ailleurs à propos de la tragédie moderne, par exemple dans un commentaire de l'Iphigénie en Aulide de Rotrou, où le sacrifice à l'autel est représenté avec une pompe particulière ${ }^{30}$ : par rapport au dénouement d'Euripide, Rotrou " change un peu la machine " pour " avoir mis le sacrifice sous les yeux du spectateur, ce qui ne laisse pas d'être un beau coup de Théatre ${ }^{31}$ ». Précisément, le jésuite, qui était dramaturge lui-même ${ }^{32}$, est particulièrement attentif à la dimension spectaculaire de la tragédie. Sur ce point, le Théâtre des Grecs illustre une orientation

Classiques Garnier, 2011. Sénèque demeurait cependant un modèle pour la rhétorique du vers dans le théâtre jésuite néo-latin, mais ici le discours esthétique de Brumoy se situe manifestement dans une autre sphère. Voir É. Flamarion, Théâtre jésuite néo-latin et antiquité. Sur le Brutus de Charles Porée (1708), Rome, École française de Rome, 2002, p. 427-458, et notamment p. 437-441, au sujet de la « fascination-répulsion » du $\mathrm{P}$. Brumoy à l'égard de Sénèque.

29 Théâtre des Grecs, t. II, p. 610-611.

30 J. Rotrou, Iphigénie, V, 3-4, éd. A. Riffaud, Théâtre complet. 2, Paris, stfm, 1999.

31 Théâtre des Grecs, t. I, "Réflexions sur Iphigénie en Aulide", p. xcvj (je souligne). L'expression " coup de Théâtre » apparaît dans l'ouvrage de Brumoy en concurrence avec " jeu de Théâtre " pour désigner les phénomènes d'animation énergique de la scène.

32 Ses pièces de théâtre composées en français pour le collège Louis-le-Grand furent imprimées : voir en particulier les tragédies Isaac et Jonathas et la pastorale Le Couronnement de David, dans le Recueil de divers ouvrages en prose et en vers par le P. Br. de la C. de J., Paris, Rollin, 1741, t. IV. 
de l'analyse esthétique qui le rapproche des Réflexions critiques de l'abbé Dubos en le distinguant des primitivistes du Grand Siècle : les Réflexions sur la poétique du P. Rapin tendaient à absorber la poétique de la tragédie dans une rhétorique tandis que Fénelon, dans la Lettre à l'Académie, invoque "spectacle " et "spectateur " sans guère envisager autre chose que la moralité et l'élocution des discours ${ }^{33}$.

Le résumé ou l'annotation des tragédies par Brumoy manifestent logiquement une inclination à imaginer une scénographie cérémonielle que le seul texte grec n'explicite pas. Dans l' Hélène d'Euripide, l'entrée de Théonoé est non seulement transcrite minutieusement dans sa dimension rituelle mais incorporée à une régie " mystérieuse " :

"L'on reconnaît la Prophétesse Théonoé à sa démarche grave et mystérieuse, et à ses paroles toutes fatidiques. Elle donne ordre à une de ses femmes de la précéder avec une lumière pour purifier l'air qu'elle va respirer, et à l'autre de passer le feu d'un flambeau sur la route pour en ôter les souillures et la profanation. Puis elle veut qu'après sa prière faite, on reporte au Palais la flamme sacrée ${ }^{34}$."

Dans Iphigénie à Aulis, la sortie de Clytemnestre au dernier acte est l'occasion d'une glose qui règle une mise en scène destinée à faire impression :

Clytemnestre. Arrêtez, cruelle; ne me quittez pas dans l'état où je suis.

IPHIGÉNIE. Je ne veux plus prolonger vos douleurs.

NOTE. Il est croyable que Clytemnestre tombe évanouie, et qu'on l'enlève dans le Palais, tandis qu'Iphigénie exhorte le Chour à chanter des hymnes en l'honneur de Diane. On devait voir au fond du Théâtre une partie de l'appareil du sacrifice : le Chøur semble en être témoin oculaire ${ }^{35}$.

Mais l'exemple le plus remarquable est constitué par les libations de la Reine dans Les Perses:

"Atossa, qui était allée chercher des libations, revient avec tous les préparatifs d'un sacrifice pour les Dieux infernaux. [...] Elle a quitté la pompe Royale ; elle est venue sans char, sans suite, sans éclat, jusqu'au lieu où elle va faire son sacrifice, tandis que le Chœur chantera des airs conformes au deuil public. Elle exhorte les Vieillards à évoquer l'ombre de Darius, pour l'interroger sur les calamités publiques. Le Chœur chante, et la Reine fait ses libations de lait, de miel, d'eau pure, de vin et d'huile, avec des fleurs. Cette cérémonie a un air tout à fait magique et théâtral. Les invocations du Chøur sont énergiques, toutes à la gloire de Darius, remplies d'idées lugubres, et composées de Strophes qui se correspondent, comme dans le chant qui a précédé. C'est là, selon toutes les apparences, tout

33 Fénelon, Lettre à l'Académie, "Projet d'un traité sur la tragédie ", éd. E. Caldarini, Genève, Droz, 1970, p. 89-99.

34 Théatre des Grecs, t. II, p. 665.

35 Ibid., t. I, «Iphigénie », p. xxxij. 
le troisième Acte, qui consiste, comme on voit, beaucoup plus en spectacle et en action, qu'en paroles ${ }^{36}$. "

Tout y est, si l'on peut dire : la dramaturgie rituelle, combinant le pathos funèbre et le prestige plastique, mais aussi ce dédain de la pompe monarchique des entrées royales. On tient là sans doute, réalisée, la synthèse de la magnificence et de la simplicité que valorisaient de façon très générale les discours liminaires. Voilà en quelque sorte l'antidote au deuil de $\mathrm{La}$ Troade sénéquienne : "Cette cérémonie a un air tout à fait magique et théâtral. " La coordination des deux adjectifs est éloquente, la seule (sauf erreur) dans tout le Théatre des Grecs. La " magie » du rite archaïque des dieux défunts semble se tourner là tout entière en " enchantement " esthétique, au sens où Brumoy lui-même souligne à propos du sacrifice d'Iphigénie qu' " il faut se prêter à l'enchantement du Théâtre ${ }^{37}$ ". Il convient d'ailleurs de se demander dans quelle mesure le qualificatif théatral n'est pas ici synonyme de tragique, comme dans la défense du sujet d'Ion ${ }^{38}$. La cérémonie conduite par la vieille reine est certes un modèle théâtral en ce qu'elle impose l'ascendant de la représentation en déjouant l'hégémonie du discours (elle " consiste beaucoup plus en spectacle et en action, qu'en paroles ») mais elle représente aussi un comble de la théâtralité tragique par cette manière extraordinaire d'esthétiser à la fois la mort et le sacré par ce rituel d'évocation.

La question qui se pose alors est celle du rapport avec la poétique de l'opéra français contemporain, qui recoupe par bien des aspects la dramaturgie du théâtre jésuite. L'esthétisation du rite funèbre, fort des appas conjugués du spectacle, de la décoration et du chant, ne regarde-t-elle pas du côté de la "tragédie en musique », ce "théâtre des enchantements " qui affectionne les scènes de sacrifice à l'autel ou d'évocations devant un tombeau ${ }^{39}$ ? Du reste, Brumoy traduisit en français le Discours sur les spectacles du P. Porée (1732), en associant expressément la tragédie déclamée et l'opéra dans un même régime poétique de la "magie » et de "l'enchantement " tout en plaidant pour une moralisation de leurs ressorts dramaturgiques ${ }^{40}$. On fera donc l'hypothèse d'une convergence persuasive entre le modèle grec et l'opéra français, lequel prétendait du

36 Ibid., t. II, p. 179.

37 Ibid., t. I, p. xcvj.

38 Ibid., t. II, p. 702 : «Je reconnais cependant avec un des plus habiles connaisseurs [le P. Porée] à qui je l'ai oui dire, que malgré les défauts réels ou apparents que j’ai cru voir dans cette Pièce, rien n'est plus véritablement Théatral, qu'une mère prête à faire mourir son fils inconnu, et à mourir inconnue par ses mains, tandis que ce double projet de parricide sert à rendre la mère au fils, et le fils à la mère " (je souligne).

39 L'évocation rituelle d'une ombre devant son tombeau figure déjà dans Ercole amante de Cavalli sur un livret de l'abbé Buti (IV, 7). Voir L. NAUDEIX, "Les ombres dans la tragédie en musique (1673-1764) ", in Fr. Lavocat et Fr. LeCercle (éd.), Dramaturgies de l'ombre, Rennes, Presses de l'université de Rennes, 2004, p. 247-261.

40 P. Brumoy, Discours sur les spectacles, éd. É. Flamarion, Toulouse, Société de Littératures Classiques, 2000, p. 21-23. 
reste recueillir l'héritage de la tragédie des Grecs. Par là s'aperçoit un glissement d'une esthétique de la cérémonie à l'autre, que plus tard dans le siècle les opéras de Gluck confirmeront à leur manière.

Restent les cas où le fonctionnement de la cérémonie dans la tragédie antique fait obstacle à sa réception moderne. Brumoy le souligne volontiers : les tragédies grecques s'inscrivent dans un contexte de coutumes et de rites propres à l'institution des cités mais qui gêne " l'intérêt " du spectateur moderne - intérêt étant à prendre d'abord au sens de l'époque, qui exprime un mode de participation émotionnelle du public, qui s'intéresse dans l'action ou pour les personnages. Que peut percevoir un spectateur moderne des paroles que Diane prononce ex machina à propos d'Hippolyte : "J'ordonne qu'en récompense de votre vertu malheureuse, Trézène vous comble d'honneurs " ?

"Ceci fait allusion aux coutumes et aux cérémonies de l'ancienne Grèce. L'on en retrouvera plusieurs autres, à mesure que l'on avancera. Ces allusions rendaient les Tragédies anciennes plus intéressantes aux spectateurs qui voyaient avec plaisir l'origine de leurs fêtes, de leurs cérémonies, et de leurs usages. Mais ce ne sont plus des traits intéressants pour nous ${ }^{41}$.»

Cet ancrage du texte tragique dans un rite circonstanciel reste cependant localisé, et le plus souvent borné aux discours de la catastrophe. Beaucoup plus intéressant est le cas de la tragédie des Bacchantes, dont la substance rituelle, déterminante pour la structure dramatique, est à la fois exaltée et marginalisée par Brumoy. Exaltée parce qu'elle illustre la tragédie grecque dans sa généalogie comme " cérémonie sacrée ", "tragédie sacrée ${ }^{42}$ "; et marginalisée pour autant que la fureur orgiaque ne saurait être accueillie dans le cadre culturel de la tragédie moderne et de ses codes, si grande est la distance entre les Grecs et les sujets de Louis XV.

"Pour accomplir sa vengeance, il [Bacchus] ordonne à une troupe de femmes étrangères qui l'entourent, (ce sont ses Prêtresses) de mener des danses Phrygiennes, avec leurs tambourins de basque, à la porte même du palais, afin d'éprouver quel sera le procédé des Thébains, à la vue de ces cérémonies en l'honneur du Dieu de la treille. [...]

Tout cela annonce une cérémonie sacrée, et confirme mon opinion sur la destination de cette pièce aux Fêtes de Bacchus, et de quelques autres à d'autres solennités. Car comme le Thêâtre devait son origine à la religion, la religion rappelait le Thêâtre à son origine. En effet, la seconde scène d'Euripide, qui est l'intermède du Chœur, n'est qu'une hymne plus que pindarique en l'honneur de Bacchus. Le choeur impose un religieux silence aux

41 Théâtre des Grecs, t. I, p. 380.

42 Ibid., t. II, p. 615: «C'est une Tragédie, et une Tragédie conduite comme celles du même auteur; mais son sujet et le tour de plusieurs scènes, me portent de plus en plus à croire que c'est une Tragédie sacrée, dont la représentation se faisait dans la joie des Fêtes de Bacchus. " 
assistants, écarte les profanes, et déclare qu'il ne veut chanter désormais que Bacchus. [...] tout retentit des noms de Denys, et de Bromien, jadis si chers au bon Ronsard. [...]

L'épode, ou le dernier couplet, est une description fort animée de la marche des Bacchantes, ou plutôt de leur course rapide [...]. Il semble qu'on les voie voler çà et là, les cheveux épars, et l'œil enflammé, dès qu'elles ont entendu la voix de leur chef ; car on y fait parler Bacchus avec un désordre que Ronsard eût mieux exprimé autrefois qu'on ne peut le faire aujourd'hui. Nous sommes en effet arrivés, ou peu s'en faut, au point où l'antiquité n'est qu'un songe $e^{43}$."

Le commentaire de Brumoy n'a plus alors qu'à osciller entre la nostalgie d'un style perdu et l'exercice d'une ironie qui ravale la transe dionysiaque à une mascarade comique. La chose est patente au sujet de la scène entre Tirésias et Cadmos :

"Malgré ces obstacles le vieux devin ne veut point de char. Il lui suffit que Bacchus et Cadmus lui servent de guide, et le nom de Bacchus seul le rajeunit. Cela est traité fort sérieusement et avec un respect très religieux. Mais il n'est pas possible d'apprivoiser nos idées avec des superstitions, qui, malgré tous nos efforts, ne peuvent nous paraître que ridicules, témoins les tournoiements des Derviches Turcs. Ceux-ci, pour être nos contemporains, n'en sont pas moins risibles sur nos Théatres, quand on y expose leurs cérémonies, comme dans Le Bourgeois Gentilhomme. Malgré la ressemblance de mille ans et de mille lieues également propres à attirer le respect du spectateur, Racine se serait bien gardé de nous peindre les superstitions des Mosquées, comme il a représenté les intrigues du Sérail. Or on doit juger des cérémonies Grecques, comme des Turques par rapport à nous. L'on passera bien celles qui ont quelque chose d'auguste, comme les sacrifices: mais pour les Orgies Bachiques, comment les passerions-nous, surtout à deux vieillards vénérables par leur âge et leur rang, qui se disposent d'un grand sérieux à danser et à courir en masques ${ }^{44}$ ?"

L'enjeu paraît consister ici en un conflit entre esthétique et religion, sous une autre forme que celle engagée par le système d'une fatalité archaïque tel qu'il peut définir la dramaturgie des tragédies antiques ${ }^{45}$. L'accommodation qui permet à Brumoy de goûter la grandeur tragique des Grecs malgré le "faux " système de leurs croyances n'a plus cours ici, pour des raisons qui sont morales certes (les orgies dionysiaques) mais non moins esthétiques : c'est la bienséance, c'est la "convenance " des caractères de la tragédie qui importe. En transgressant la pureté du decorum, la cérémonie bascule irrémédiablement dans le burlesque. Brumoy ironise de même à propos de la scène où Tirésias s'adresse à Penthée afin de justifier le rite dionysiaque :

43 Ibid., p. 614-615 (je souligne).

44 Ibid., p. 616 (je souligne).

45 Voir les importantes réflexions de J.-M. VAlEnTin, " "Un système [...] faux [...], mais d'où résultent de si grandes beautés". Tragédie, tragique et destin dans le drame de tradition catholique : le Théâtre des Grecs de Pierre Brumoy s.j. ", in L'École, la ville, la cour, Paris, Klincksieck, 2004, p. 173-191. 
"Le devin, pour ne rien omettre de ce qui peut relever les cérémonies des Bacchanales, ajoute que la fureur qu'on y puise est prophétique, et que le vin dévoile l'avenir. Il est beaucoup plus vrai qu'il découvre le présent et le passé suivant le proverbe in vino veritas $^{46}$."

Quant au récit du Berger, il ne jette pas seulement le doute sur la gravité de la tragédie grecque, mais permet de vérifier des analogies inattendues avec les genres modernes :

" "Car sans le vin, ajoute-t-il, plus d'amour, plus de joie." Ce mot scandaleux fait bien voir que la piété Grecque n'était pas toujours fort sévère en fait de morale, et que l'impiété de Penthée avait quelque chose de plus vertueux. Cela me ferait presque penser que le caractère de cette Tragédie est celui d'un véritable Opéra, et qu'il y en a eu apparemment plus d'un modèle dans l'antiquité. En effet, on voit tant de rapport entre nos Opéras et cette Pièce, que cela seul pourrait servir à justifier ma pensée ; et d'ailleurs quel inconvénient y aurait-il à croire que la Tragédie et l'Opéra eussent pris naissance en même temps dans les hymnes composées en l'honneur de Bacchus? cette antiquité de l'Opéra ne le rendra pas plus innocent à nos yeux, sur tout tant que le vice paré des plus brillantes couleurs y triomphera impunément de la vertu ${ }^{47}$. "

La référence à l'opéra apparaît désormais explicitement, mais à propos de l'immoralité d'une doxa hédoniste, celle-là même que Brumoy reproche, après Porée et de façon conventionnelle, aux "maximes " chantées à l'opéra ${ }^{48}$. Pourtant on se demandera si ce « rapport " entre la tragédie grecque et l'opéra français, point crucial dans les débats sur la " tragédie en musique " dès l'origine du genre, n’intéressait pas également, et sérieusement, la transposition d'une fureur expansive en cérémonial esthétique. Après tout, le sujet des Bacchantes avait été traité en opéra à sujet horrible dès 1703, avec ce Penthée, dont le livret dû à La Fare avait été mis en musique par Philippe d'Orléans, futur Régent ${ }^{49}$.

Catégorie critique en ce qu'elle permet de distinguer Sénèque d'Euripide et Racine d'Eschyle, la cérémonie inhérente à la tragédie des Grecs importe aussi dans la mesure où elle constitue un point de tension interne à la poétique proposée par Brumoy. Vouée

46 Théâtre des Grecs, t. II, p. 618.

47 Ibid., p. 625.

48 P. Brumoy, Discours sur les spectacles, p. 55-61.

49 Voir J.-P. Montagnier, Un mécène musicien : Philippe d'Orléans, Régent, Paris, Zurfluh, 1996, p. 74-80. Le livret figure dans les Poésies de Monsieur le Marquis de La Fare, Amsterdam, Bernard, 1755, p. 223-276. 
à se fondre dans le naturel de l'expression pathétique, elle affiche cependant l'artifice d'un rite dont la valeur ne saurait plus être qu'esthétique. Dès les discours liminaires, Brumoy vantait dans le chœur antique un mouvement scénique "fort mystérieux ", qu'il compare au tournoiement des derviches, mais pour en résorber la valeur sacrale dans « l'agrément et la splendeur des spectacles ${ }^{50}$ ». Ce faisant, le caractère incantatoire de la cérémonie dans la dramaturgie est gommé au profit de sa valeur plastique et scénique. Brumoy est sur ce point cohérent, puisqu'il considère que les chœurs " donnent beaucoup plus aux yeux qu'aux oreilles ${ }^{51}$ ". Dès lors, comme la " magie ", comme l'" enchantement ", les «mystères » défunts de la cérémonie passent désormais du côté du plaisir thêâtral. L'importance même du terme cérémonie dans le vocabulaire critique du jésuite signale une esthétisation du sacré dont la tragédie en musique offre à l'époque, de fait, la réalisation théâtrale la plus accomplie. L'analyse des Bacchantes atteste ce paradoxe : c'est par sa parenté avec le genre moderne de l'opéra que la " tragédie sacrée " des Anciens pouvait combler la distance qui l'éloignait d'un spectateur de 1730. Objet de délectation, la dramaturgie cérémonielle demeurait au même moment combattue théoriquement par l'impératif de la simple nature, à l'image du jeu des acteurs antique dont Brumoy affirme sans sourciller : "Tout cet ensemble [...] était trop machinal et n'avait point le naturel de l'action toute nue ${ }^{52}$.»

Jean-Philippe GrosPerRIN

Université de Toulouse (UTM)

PLH-ERASME

Pavillon de la Recherche

5 allées $A$. Machado

F-31058 Toulouse Cedex 9

grosperr@univ-tlse2.fr

50 Théâtre des Grecs, "Discours sur l'origine de la tragédie », XV, t. I, p. lxxviij-lxxix.

51 Ibid., t. I, p. lxxv.

52 Ibid., XVIII, t. I, p. xcviij. 